\title{
All-Photonic Molecular Keypad Lock
}

\author{
Joakim Andréasson, ${ }^{*[a]}$ Stephen D. Straight, ${ }^{[b]}$ Thomas A. Moore, ${ }^{*[b]}$ Ana L. Moore, ${ }^{*[b]}$ and \\ Devens Gust* ${ }^{[b]}$
}

Herein we demonstrate a molecular triad (1, Figure 1) comprising two photochromic units and a fluorescent porphyrin reporter that functions as an all-photonic keypad lock. The lock is a molecular automaton that opens only when exposed to one out of 8 possible 2-digit codes, can be reset many times, and can be activated remotely.

Investigations of molecular approaches to logic operations began with simple switches and logic gates, and is now focused on the design and the synthesis of more complex molecular devices including small electronic circuits. ${ }^{[1-13]}$ An example is the molecular keypad lock. Although molecular keypad locks and similar devices have been demonstrated before ${ }^{[14-18]}$ the previous examples all require addition of chemical species as input signals. This imposes limitations on both the operational speed and the nature of the surrounding medium, as fluid solution is required. Moreover, the repeated operation of chemically driven devices leads to build-up of chemical waste and/or dilution. Moleculebased logic elements with optical inputs and outputs such as $\mathbf{1}$ and other molecules we have reported ${ }^{[1-4,8]}$ do not require access for chemicals or wires, can in principle operate on a much faster time scale and in rigid media, and can be cycled numerous times with no buildup of byproducts. A special advantage of optical inputs for the keypad lock is that it is capable of being operated remotely by light beams.

The functional principle of a keypad lock is that it gives an output signal (opens) only when given the correct inputs in the correct order. In logic terms, a 2-input keypad lock such as $\mathbf{1}$ corresponds to a 2-input priority AND (PAND) gate. The major difference between the PAND gate and the regular AND gate is that its output is sensitive not only to the application of the inputs but also to the sequence in which they are applied. The 2-input

[a] Prof. Dr. J. Andréasson

Department of Chemical and Biological Engineering, Physical Chemistry Chalmers University of Technology

41296 Göteborg (Sweden)

Fax: (+46) 31-772 3858

E-mail: a-son@chalmers.se

[b] Dr. S. D. Straight, Prof. Dr. T. A. Moore, Prof. Dr. A. L. Moore, Prof. Dr. D. Gust

Department of Chemistry and Biochemistry

Arizona State University

Tempe, AZ 85287 (USA)

Fax: (+1) 480-965-5927

E-mail: gust@asu.edu
PAND gate has two two-valued (on, off) inputs, $A$ and $B$, and an output. Any of the 6 ordered combinations of inputs in which at least one input is off leaves the output off. When both inputs are on, the only application sequence that yields an on output is $A$ followed by $B$. In addition to the keypad lock, PAND gates have applications in fault analysis and elsewhere.

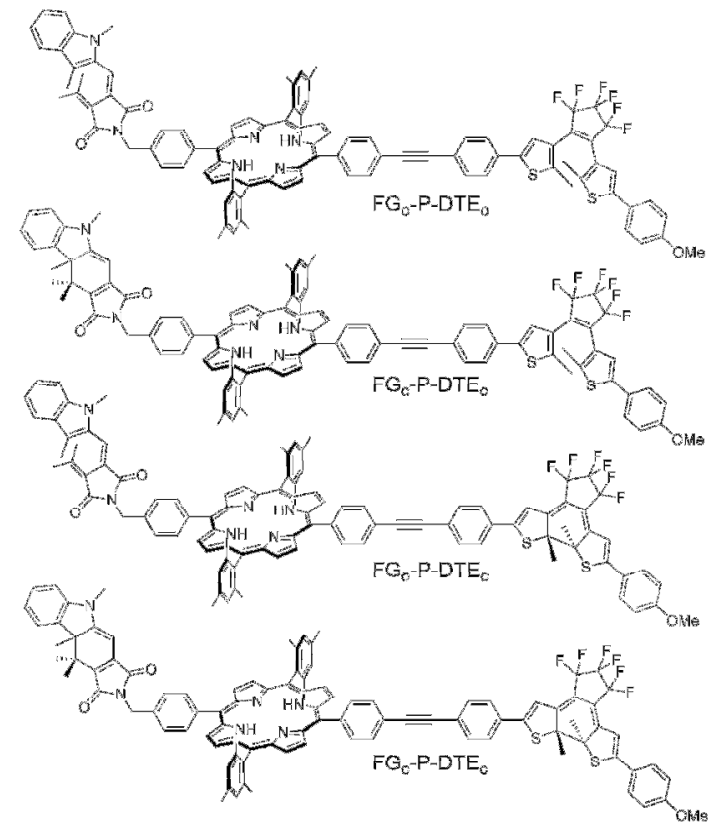

Figure 1. Photoisomers of triad 1

The synthesis, structure, and photophysical properties of triad $\mathbf{1}$ have been described previously. ${ }^{[19]}$ Briefly, the triad consists of two individual photochromic units, one from the fulgimide (FG) and the other from the dithienylethene (DTE) families, covalently linked to a central tetraarylporphyrin (P). Each photochromic unit may exist in two isomeric forms that are interconverted by light of different wavelengths. The open, colorless forms of both photochromes (FGo and DTEo) are isomerized to the colored, closed forms (FGc and DTEc) by exposure to UV light (e.g. 366 $\mathrm{nm}$ ), whereas the reverse reactions are induced by visible light. Each photochrome may be selectively opened or closed without significantly affecting the state of the other photochrome by using suitable combinations of wavelength and light dose. Thus, it is possible to prepare solutions of $\mathbf{1}$ highly enriched in each one of 
the four possible isomeric forms. For the keypad function, only three of these isomers are required: FGo-P-DTEo, FGc-P-DTEo, and FGc-P-DTEc. The absorption spectra of solutions highly enriched in the three forms are shown in Figure 2, and the conditions used to obtain them appear in Scheme 1.

The two photochromes and the porphyrin of $\mathbf{1}$ interact photophysically. For example, when the molecule is excited at $470 \mathrm{~nm}$, light is absorbed mainly by FGc, when present, as the extinction coefficients for the porphyrin and the other isomeric forms of the photochromes are very small at this wavelength. The first excited singlet state of FGc thus formed is strongly quenched due to efficient singlet-singlet energy transfer to the porphyrin. ${ }^{[19]}$ The porphyrin excited state is fluorescent (Figure 3), but when DTE is present in the closed form, DTEc strongly quenches porphyrin emission by singlet-singlet energy transfer. Excitation of FGc is relayed to DTEc via the porphyrin intermediary. DTEo cannot quench the porphyrin excited state due to the lack of spectral overlap between the porphyrin emission and the absorption of DTEo. Hence, the prerequisites for strong porphyrin emission are that the FG photochrome must be in the closed form to absorb the $470 \mathrm{~nm}$ light and act as a sensitizer for the porphyrin, and also that the DTE photochrome must be in the open form to prevent quenching of the porphyrin emission: FGc-P-DTEo is the only form where the porphyrin reporter displays strong emission upon excitation of the triad at $470 \mathrm{~nm}$. The relative porphyrin emission intensities in the different forms of the triad are shown in Figure 3.

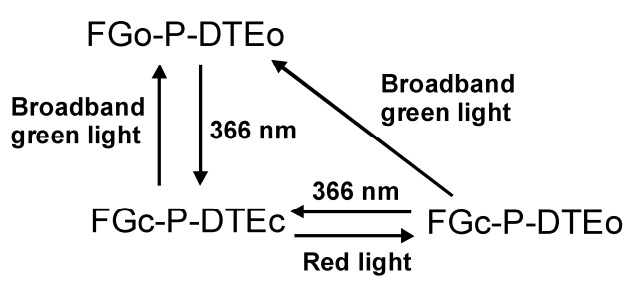

Scheme 1. Interconversion pathways of triad $\mathbf{1}$ that are relevant for the performance of the keypad lock.

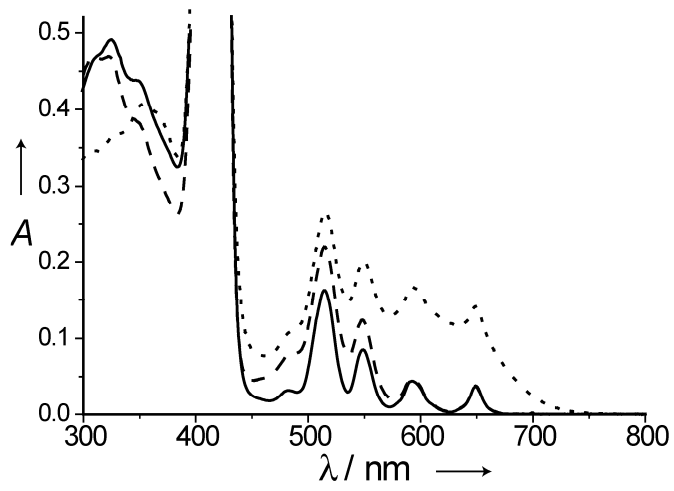

Figure 2. Absorption spectra of triad $\mathbf{1}$ in 2-methyltetrahydrofuran: FGo-P-DTEo $(-)$, FGc-P-DTEo $(---)$, and FGc-P-DTEc $(\bullet \bullet)$.

The characteristics of triad $\mathbf{1}$ described above make it possible to realize the all-photonic molecular keypad lock. The function is based on the capacity of the triad to perform as a two-input PAND gate with reset capacity. Before application of inputs, the triad is initially set to the FGo-P-DTEo form using broadband green light (460 $\mathrm{nm}<\lambda<590 \mathrm{~nm}$ ). Input $A$ is UV light at $366 \mathrm{~nm}$, and input
$B$ is red light $(\lambda>580 \mathrm{~nm})$. The output is porphyrin emission excited at $470 \mathrm{~nm}$ and monitored at $650 \mathrm{~nm}$. In the initial FGo-PDTEo state, the triad does not absorb significantly at $470 \mathrm{~nm}$, and the weak emission intensity shown in Figure 3 stems from direct excitation of the porphyrin unit. With a proper choice of threshold level (e.g. the horizontal line in Figure 3) the gate output is off. Exposing FGo-P-DTEo to red light for 15 min (input $B$ ) results in no isomerization because neither photochrome in the open form absorbs at this wavelength, and the gate output remains off. Exposing the sample to $366 \mathrm{~nm}$ UV light for 2 min (input A) isomerizes FGo-P-DTEo to FGc-P-DTEc. This form also displays only weak fluorescence and the gate output remains off (Figure 3). Thus, none of the 6 combinations of ordered inputs in which one input is off yields an on output.

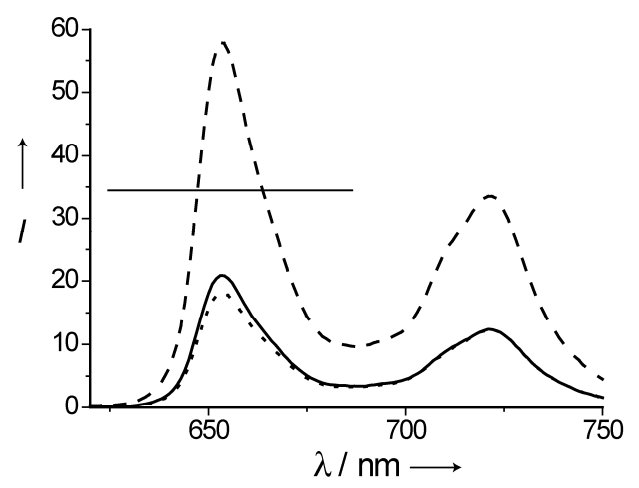

Figure 3. Fluorescence emission spectra of triad 1 in 2-methyltetrahydrofuran upon excitation at $470 \mathrm{~nm}$ : FGo-P-DTEo (-), FGc-P-DTEo (- - -), and FGc-P-DTEc $(\bullet \bullet)$. The horizontal line represents a threshold level for the readout process.
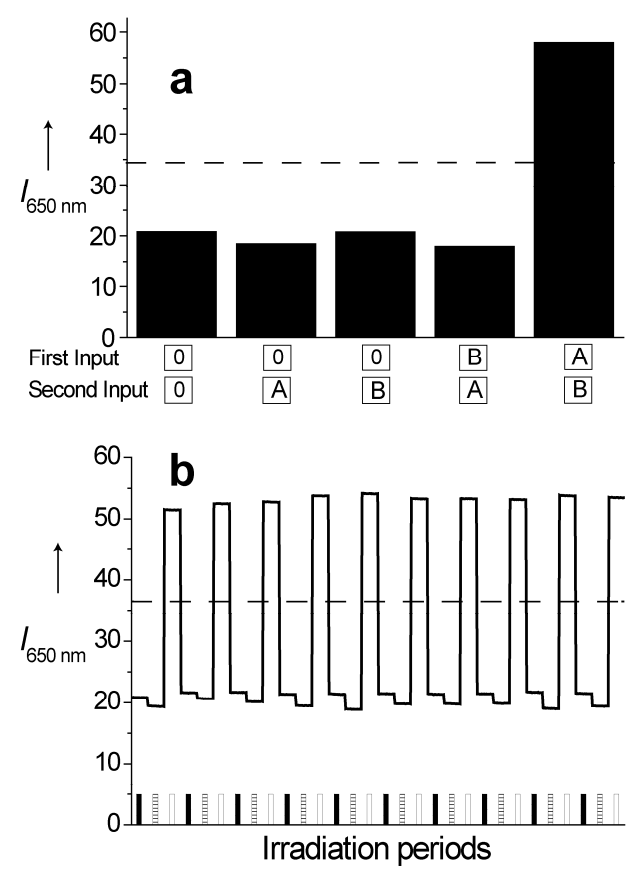

Figure 4. a) Fluorescence emission intensities (arbitrary units) at $650 \mathrm{~nm}$ upon excitation at $470 \mathrm{~nm}$ following various input sequences $(0$, no input on; A, input $A$ on, $\mathrm{B}$, input $B$ on). Prior to applying the inputs, 1 was reset to the FGo-P-DTEo form using broadband green light. Reversing the order of application in the first three columns yields identical results. b) Photocycling of 1. Emission intensities at $650 \mathrm{~nm}$ (excitation at $470 \mathrm{~nm}$ ) following sequential irradiation using broadband green light (reset, black bars), $366 \mathrm{~nm} \mathrm{UV} \mathrm{light} \mathrm{(input} A$, striped bars), and red light (input $B$, white bars). The small variations in height of each emission time-trace show the variation in signal amplitude during measurement for $5 \mathrm{~s}$. The dashed lines in both Figures are thresholds used to distinguish an on output from an off output. 
Turning now to the situation when both inputs are turned on, exposure to $B$ followed by exposure to $A$ will convert the initial FGo-P-DTEo to FGc-P-DTEc, which displays only weak fluorescence, leaving the gate output off. Finally, exposing FGoP-DTEo to input $A$ will convert it to FGc-P-DTEc, and then applying input $B$ to this isomer will generate FGc-P-DTEo, which is strongly fluorescent. The output is now on, the keypad lock has opened, and the conditions for a 2-input PAND gate have been satisfied.

Figure $4 \mathrm{a}$ shows the actual operation of the keypad lock. The porphyrin emission intensity at $650 \mathrm{~nm}$ upon exposure to the various 2 -input sequences followed by readout using excitation at $470 \mathrm{~nm}$ is depicted. For the first three input combinations, reversing the order of application yields the identical result. With a proper choice of threshold level, the emission intensity is high enough to open the lock only upon the $A$ followed by $B$ input sequence.

This kepad lock opens only when one of eight possible input combinations is applied. The number of input combinations could be increased by including an additional input signal, input $C$, corresponding to the resetting of the triad to the initial FGo-PDTEo form using broadband green light for $12 \mathrm{~min}$. As is seen in Scheme 1, broadband green light will isomerize any form of the triad to FGo-P-DTEo. Using a 10-key keypad consisting of 8 reset keys (input $C$ ) together with inputs $A$ and $B$ would give a total of 100 possible 2-digit codes, only one of which would open the lock. Used in this way, any entry of a 2-digit code would be followed by a readout operation using $470 \mathrm{~nm}$ excitation and a reset operation with green light.

As noted above, the possibility of resetting the keypad lock with broadband green light makes it possible to use the lock numerous times without addition or removal of chemical species. For this to be useful, the photodecomposition of the triad must be low to enable repeated cycling through the various isomeric forms. Figure $4 \mathrm{~b}$ shows that after 10 cycles of broadband green, $366 \mathrm{~nm}$, and red light there are no signs of sample degradation. The associated signal noise is shown as the minute variation in height of each bar and is clearly too small to interfere with device operation.

In summary, we have shown proof-of-principle of a molecular all-photonic 2-input keypad lock with reset capacity. The lock is capable of distinguishing one 2-digit code out of 8 possible combinations. The lock is operated by five optical signals in the $\mathrm{UV} /$ vis region including fluorescence emission, which signals the opening of the lock. The lock can be used numerous times without any photodegradation affecting the performance.

\section{Experimental Section}

The synthesis and characterization of triad 1 have been reported. ${ }^{[19]}$ Distilled 2methyltetrahydrofuran was used as the solvent for spectroscopic measurements. The samples were degassed by six freeze-pump-thaw cycles to a final pressure of approximately 10-5 Torr. The absorption measurements were performed using a CARY 4000 UV-vis spectrometer. A SPEX Fluorolog $\tau 2$ was used for the emission measurements. The sample concentration was $\sim 7.5 \mathrm{mM}$. The broadband green light and the red light were generated by a $1000-\mathrm{W} \mathrm{Xe/Hg} \mathrm{lamp} \mathrm{running} \mathrm{at} 450 \mathrm{~W}$. The light from the $\mathrm{Xe} / \mathrm{Hg}$ lamp was filtered by two hot mirrors (each with $A=1.8$ at 900 $\mathrm{nm})$ to reduce the IR intensity. A VG 9 glass filter $(A>1.5$ between 460 and 590 $\mathrm{nm})$ was used for the broadband green light, and a long-pass filter $(\lambda>580 \mathrm{~nm})$ was used for the red light. The resulting light power densities were $\sim 9 \mathrm{mWcm}^{-2}$ and $\sim 50$ $\mathrm{mWcm}^{-2}$ for the broadband green light and the red light, respectively. A UVP UV lamp (Model UVGL-25, $\sim 2 \mathrm{mWcm}^{-2}$ ) was used to provide the $366 \mathrm{~nm}$ UV light.
With the use of the broadband green light and the red light, only one-third of the sample volume was exposed to the light at any one time, whereas the whole sample volume was exposed to $366 \mathrm{~nm}$ light. The samples were continuously stirred during all irradiation processes.

\section{Acknowledgements}

This work was supported by the Swedish Research Council (VR), the European Research Council (ERC FP7/2007-2013 No. 203952) and the U.S. National Science Foundation (CHE-0352599).

\section{Keywords: photochromism • photochem. react. •} porphyrins $\bullet$ chromophores

[1] J. Andréasson, S. D. Straight, S. Bandyopadhyay, R. H. Mitchell, T. A. Moore, A. L. Moore and D. Gust, J. Phys. Chem. C 2007, 111, 14274-14278.

[2] J. Andréasson, S. D. Straight, S. Bandyopadhyay, R. H. Mitchell, T. A. Moore, A. L. Moore and D. Gust, Angew. Chem. Int. Ed. 2007, 46, 958-961.

[3] J. Andréasson, S. D. Straight, G. Kodis, C. D. Park, M. Hambourger, M. Gervaldo, B. Albinsson, T. A. Moore, A. L. Moore and D. Gust, J. Am. Chem. Soc. 2006, 128, 16259-16265.

[4] J. Andréasson, S. D. Straight, T. A. Moore, A. L. Moore and D. Gust, J. Am. Chem. Soc. 2008, 130, 11122-11128.

[5] V. Balzani, A. Credi and M. Venturi, ChemPhysChem 2003, 4, 49-59.

[6] A. P. de Silva and N. D. McClenaghan, Chem. Eur. J. 2004, 10, 574-586.

[7] B. M. Frezza, S. L. Cockroft and M. R. Ghadiri, J. Am. Chem. Soc. 2007, 129, 14875-14879.

[8] D. Gust, T. A. Moore and A. L. Moore, Chem. Comm. 2006, 1169-1178.

[9] Y. Liu, W. Jiang, H. Y. Zhang and C. J. Li, J. Phys. Chem. B 2006, 110, 14231-14235.

[10] T. Niazov, R. Baron, E. Katz, O. Lioubashevski and I. Willner, Proc. Natl. Acad. Sci. USA 2006, 103, 17160-17163.

[11] E. Perez-Inestrosa, J. M. Montenegro, D. Collado and R. Suau, Chem. Comm. 2008, 1085-1087.

[12] U. Pischel, Angew. Chem. Int. Ed. 2007, 46, 4026-4040.

[13] F. M. Raymo, Adv. Mater. 2002, 14, 401-414.

[14] Z. Q. Guo, W. H. Zhu, L. J. Shen and H. Tian, Angew. Chem. Int. Ed. 2007, $46,5549-5553$

[15] D. Margulies, C. E. Felder, G. Melman and A. Shanzer, J. Am. Chem. Soc. 2007, 129, 347-354.

[16] G. Strack, M. Ornatska, M. Pita and E. Katz, J. Am. Chem. Soc. 2008, 130, 4234-4235.

[17] W. Sun, C. Zhou, C. H. Xu, C. J. Fang, C. Zhang, Z. X. Li and C. H. Yan, Chemistry-A European Journal 2008, 14, 6342-6351.

[18] M. Suresh, A. Ghosh and A. Das, Chem. Comm. 2008, 3906-3908.

[19] S. D. Straight, P. A. Liddell, Y. Terazono, T. A. Moore, A. L. Moore and D. Gust, Adv. Funct. Mater. 2007, 17, 777-785.

Received: ((will be filled in by the editorial staff))

Revised: ((will be filled in by the editorial staff)) Published online: ((will be filled in by the editorial staff)) 


\section{Entry for the Table of Contents}

Off and $O n$

Joakim Andréasson, * Stephen D.

Straight, Thomas A. Moore, * Ana

L. Moore, * and Devens Gust* Page

- Page

\section{All-Photonic Molecular Keypad Lock}

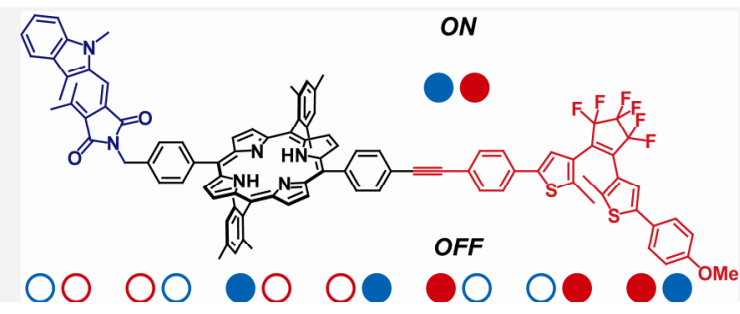

Off and On: A molecular triad consisting of a porphyrin linked to two different, independently addressable photochromic moieties functions as a molecular keypad lock with all photonic inputs and output. The porphyrin correlates the responses of the two inputs to light of different wavelengths and provides an appropriate output as fluorescence. The molecule gives a fluorescence output signal only when one of 8 possible input combinations has been applied. Using a 10-key keypad, the lock would open only upon receipt of one of 100 possible 2-digit codes. 